\title{
Operationsbericht Shuntanlage Vom Traum zur Wirklichkeit?
}

Ein wichtiger Bereich jeder Operation ist die Anfertigung eines Operationsberichtes. Dieser enthält alle im Zusammenhang mit der Operation notwendigen Fakten. Der Operationsbericht ist eine der Grundlagen für die Planung des weiteren Handelns wie etwa die Punktion, die Bewertung der Fluss- und Venenentwicklung oder der Langzeitprognose einer arteriovenösen Fistel. Trotz der Pflicht, einen Operationsbericht zu erstellen, ist nicht sichergestellt, dass dieser nach stationären Aufenthalten mit dem Entlassungsbrief in das ambulante Dialysezentrum weitergeleitet wird.

In der Disziplin der Shuntchirurgie liegt eine Ausnahmesituation vor, da die Anatomie, Morphologie und zu erwartende Prognose der AV-Fisteln (AV: arteriovenös) sehr variabel ist. Außerdem variieren die Inhalte der OP-Berichte (OP: Operation) unter dem Aspekt „Was muss der Kunde (Nephrologe, Pflegepersonal) wissen?"“ erheblich. Dies waren die Beweggründe für einen retrospektiven, systematischen Vergleich der Inhalte unserer archivierten OP-Berichte. Es wurde nicht zwischen Erstanlagen oder Revisionen unterschieden. Unser ambulantes Dialysezentrum betreute zum Zeitpunkt der Erhebung 145 Patienten in der LC- ("Limited-Care") oder Praxisdialyse.

\section{Besondere Aspekte des \\ OP-Berichtes und deren Aussage}

Neben den allgemeinen Inhalten spielen folgende Besonderheiten (1) eine wichtige Rolle, die ich in den von mir gesichteten OP-Berichten verglich:

- vorgefundener Situs

- pathologische Veränderungen

- morphologische Veränderungen

- Prognose und Revisionsmöglichkeit

- postoperatives Shuntrauschen

- Punktionshinweis
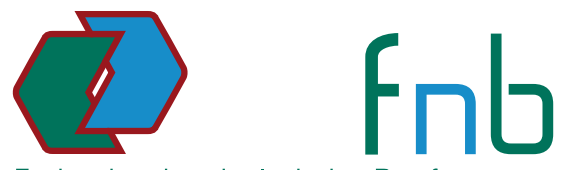

Fachverband nephrologischer Berufsgruppen

Geschäftsstelle fnb

Uschi Gasper

In den Beunen 6, 65479 Raunheim

Tel.: 06142/408549

Fax: $06142 / 408551$

E-Mail: uschi.gaspar@nephro.fachverband.de
Der vorgefundene Status gibt Information über die Lage, Größe und Durchmesser der verwendeten Gefäße. Arterien sollten nicht kleiner als $2 \mathrm{~mm}$ sein und die Vene sollte einen Durchmesser von mehr als $4 \mathrm{~mm}$ aufweisen.

Pathologische Veränderungen beeinflussen die zu erwartende Prognose, wie etwa die Mediasklerose der Arterien. Diese Arterie wird nur bedingt einen ausreichenden Fluss für den Shunt anbieten können, da sie nicht in der Lage ist, sich zu erweitern. Aufgrund der Vielfachpunktion durch Blutabnahmen sklerosieren diese Venensegmente und neigen zu Stenosen.

Morphologische Veränderungen sind etwa ein Stealsyndrom oder eine Kollateralbildung der Vene. Dieser Hinweis ist wichtig, um diese Veränderungen auch klinisch bewerten zu können.

Ein Hinweis zur Prognose bzw. eine Empfehlung bezüglich der Revision ist wichtig, um bei insuffizienter Shuntentwicklung frühzeitig reagieren und alle folgenden Maßnahmen planen zu können. Ist eine chirurgische Revision in diesem Fall sinnvoll? Sollte eine radiologische Intervention vorgezogen werden, um einige Monate gewinnen $\mathrm{zu}$ können?

Das postoperative Shuntgeräusch wird meist mit „nach Wundverschluss gleichmäßiges Shuntrauschen vorhanden“ angegeben.

Der Punktionshinweis ist insbesondere für uns Pflegekräfte von Bedeutung. Patchplastiken als Revisionsmethode können frühestens nach abgeschlossener Wundheilung im Wundbereich punktiert werden. Bei der Erstpunktion nach einer Neuanlage sind Aussagen über den Venendurchmesser und die Venentiefe wichtig für die Nadelwahl und Punktionstechnik.

\section{Ergebnisse}

In 7 verschiedenen Einrichtungen im Umkreis von zirka $150 \mathrm{~km}$ wird der AVShunt für die Patienten unseres Dialysezentrums angelegt. Vier von 7 Kliniken konnten in den Vergleich einbezogen werden, da ich als Datengrundlage pro Klinik mindestens 10 OP-Berichte als Mindestmaß ansetzte.

Der vorgefundene Situs sowie pathologische Veränderungen wurden nur von einer Klinik in allen OP-Berichten generell beschrieben. Morphologische Veränderungen nannten nur 2 von 4 Kliniken. Die Prognose sowie eine Revisionsempfehlung suchte ich bei 3 Kliniken vergeblich. Erstaunlicherweise gaben alle 4 Kliniken das freie Shuntrauschen nach dem Nahtverschluss an. Keine der Beschreibungen differenzierte dies aber qualitativ in systolisches und diastolisches Rauschen (2).

\section{Fazit}

Der OP-Bericht mit den besonderen Aspekten der AV-Shuntanlage sollte standardisiert werden. Fachverbände wie die „Vascular Access Society“, der fnb und Ärzteverbände wie die Deutsche Arbeitsgemeinschaft für Klinische Nephrologie (DAGKN) und die Deutsche Gesellschaft für Fachkrankenpflege (DGF) sollten gemeinsam definieren, welche Inhalte in einem OP-Bericht nach einer Shuntanlage oder Revision zu nennen sind.

„Vom Traum in die Wirklichkeit“ - wir können dieses Motto mit Leben füllen, indem wir die besonderen Aspekte des OP-Berichtes zum Thema gemeinsamer regionaler Fortbildungsveranstaltungen machen.

Beate Spindler, Marbach

\section{Literatur}

1 Krönung G. Der Operationsbericht, Dialyse aktuell 2006; 10: 32-37

2 Hollenbeck M. Klinische Untersuchung der nativen arteriovenösen Dialysefistel. Vascular Access Forum Newsletter 2005; 2 